\title{
Review \\ The Emerging Neurobiology of Bipolar Disorder
}

Paul J. Harrison, ${ }^{1,2, \star}$ John R. Geddes, ${ }^{1,2}$ and Elizabeth M. Tunbridge ${ }^{1,2}$

Bipolar disorder (BD) is a leading cause of global disability. Its biological basis is unknown, and its treatment unsatisfactory. Here, we review two recent areas of progress. First, the discovery of risk genes and their implications, with a focus on voltage-gated calcium channels as part of the disease process and as a drug target. Second, facilitated by new technologies, it is increasingly apparent that the bipolar phenotype is more complex and nuanced than simply one of recurring manic and depressive episodes. One such feature is persistent mood instability, and efforts are underway to understand its mechanisms and its therapeutic potential. BD illustrates how psychiatry is being transformed by contemporary neuroscience, genomics, and digital approaches.

\section{Bipolar Disorder and the New Psychiatry}

Psychiatry still relies largely on 19th-Century diagnostic categories. These are based on clusters of symptoms rather than biological markers, and are treated with drugs discovered serendipitously several decades ago. BD typifies this unsatisfactory state of affairs. Although its name has changed [it was formerly known as manic depression (see Glossary)], its cardinal features, and how it is assessed and treated (Box 1) have barely altered. An important reason for this stagnation has been the lack of any real traction on its causes and underlying biology, beyond its well-established high heritability [1]. Although there is evidence for altered structural and functional brain connectivity [2-4], and changes in markers of oxidative stress [5], mitochondrial function [6], inflammation [7], circadian rhythms [8], and dopamine [9], it remains difficult to integrate these diverse findings, and to disentangle causative changes from those that are secondary to the disorder and its treatment.

The situation is belatedly improving. While optimism must be tempered by appreciation of the many complexities, there are realistic prospects for a transformation in our understanding of BD and how it is diagnosed and treated. Here, we highlight two areas of current interest: the discovery of the first BD risk genes and their implications, and the application of novel technologies with the potential to refine, or redefine, the BD phenotype. These developments exemplify how genomics, neuroscience, and digital technologies are heralding a new era for psychiatry. For broader reviews of $\mathrm{BD}$, see $[10,11]$.

\section{The Genomics of Bipolar Disorder}

A child of an affected parent has about a tenfold increased risk of developing BD, and twin studies estimate a heritability of 0.7-0.8 [1]. There is no evidence for Mendelian inheritance or for genes of major effect. Instead, as with most psychiatric disorders, there are multiple susceptibility loci, each of small effect, which genome-wide association studies (GWAS) are beginning to identify. Several GWAS, and meta-analyses thereof, have been carried out since 2007; Table 1 lists the loci and implicated genes that have emerged to date. The combined

\section{Trends}

$\mathrm{BD}$ is highly heritable and mostly attributable to common variants of small effect. Several risk genes and gene networks have been identified.

Calcium signalling is prominent among the genetic risk pathways, and currently appears to have the greatest therapeutic traction.

Digital technologies and sophisticated mathematical and computational analyses are being used to quantify and understand $\mathrm{BD}$.

These new methods reflect, and are promoting, reconceptualisation of $\mathrm{BD}$ as a chronic instability of mood and neural circuitry.

Stem cells are becoming an integral part of the approaches to understanding $\mathrm{BD}$ and its pharmacotherapy.

New experimental medicine models are being applied to identify and rapidly test potential mood-stabilising treatments.

${ }^{1}$ Department of Psychiatry, University of Oxford, Warneford Hospital, Oxford, OX3 7JX, UK

${ }^{2}$ Oxford Health NHS Foundation Trust, Warneford Hospital, Oxford, OX3 7JX, UK

${ }^{*}$ Correspondence:

paul.harrison@psych.ox.ac.uk (P.J. Harrison). 


\section{Box 1. Bipolar Disorder: A Clinical Primer}

The classic picture of $\mathrm{BD}$ is like a modified sine wave, with mood fluctuating between episodes of mood elevation (mania) and depression, interspersed with periods of euthymia. The number of episodes in each mood phase, and their duration, varies markedly between individuals, but for most patients the depressive episodes are more prolonged and are responsible for much of the morbidity of the disorder.

The depressive episodes of $\mathrm{BD}$ are broadly similar in nature and severity to those of 'ordinary' depression. A manic episode includes not only significant elevation of mood, but also related changes in behaviour, such as a reduced need for sleep, increased energy, grandiose thoughts and beliefs, rapid speech, increased libido, and reckless behaviour (e. g., spending excessively). In severe episodes, psychotic symptoms (delusions and hallucinations) may also be present; for example, the person may believe, or hear voices telling them, that they have superpowers (and they may then act accordingly). 'Hypomania' refers to a milder and less prolonged form of mania. The exact criteria depend upon the classificatory system used (ICD-10 or DSM-5); the latter subdivides BD into bipolar I and bipolar II. Although not part of the diagnostic criteria, cognitive impairment is a notable aspect of $\mathrm{BD}$; it is present at first episode and persists during euthymia. Attention, processing speed, and verbal learning and fluency are the domains most affected. At least half of patients with $\mathrm{BD}$ also have an anxiety disorder or substance use disorder. Patients are typically diagnosed during their 20 s, following a long prodrome, and actual onset is often in adolescence. The lifetime prevalence of BD is approximately $1 \%$, rising to $4 \%$ if a broader definition of bipolar spectrum disorder is used. The major risk factors are genetic (see the main text), but environmental factors, including childhood adversity, also have a role. Of patients with BD, $10 \%$ die by suicide and this, coupled with an excess mortality from natural causes, shortens average life expectancy by approximately 15 years.

The mainstay of $\mathrm{BD}$ treatment is pharmacological, with lithium salts or the antiepileptic drug sodium valproate used for prophylaxis. Antipsychotics, antidepressants, and antiepileptic drugs are given to treat the mood episodes. Psychoeducation and psychological treatments also have an important supporting role. All current treatments have limited efficacy, and the drugs can have serious adverse effects.

For an introduction to clinical aspects of $\mathrm{BD}$, see $[10,95]$.

sample sizes remain small by GWAS standards, and more loci remain to be identified; indeed, the forthcoming Psychiatric Genomics Consortium analysis, comprising over 20000 BD cases and 30000 controls, identifies 19 significant loci, including 12 novel ones. Initial exome and genome sequencing data suggest that rare deleterious variants also have a role in some BD cases, but their identity and overall contribution to the disorder remain unclear [12-14]. Within $\mathrm{BD}$, there is modest clinicogenetic heterogeneity, for example, based on the predominant symptoms, or between bipolar I and bipolar II subtypes [15]. However, there is little evidence for BD-specific genes; joint GWAS analyses show substantial commonalities in risk loci for BD and schizophrenia [16], as well as significant overlap with other major psychiatric disorders [17] and with intermediate phenotypes, including circadian traits $[18,19]$. One distinction between schizophrenia and BD is that copy number variation is much less prominent in the latter [20].

Although the genomics of $\mathrm{BD}$ are in their infancy, efforts have begun to understand the biological basis for the associations identified to date. Interest has centred on two genes (CACNA1C and ANK3) because of what was already known of their functions. CACNA1C is discussed in detail below. ANK3 encodes ankyrin G, which couples axonal voltage-gated sodium channels to the cytoskeleton and also has roles in dendrites and glia; another risk gene, TRANK1, contains multiple ankyrin repeat domains, suggesting some shared functions. Complementing the focus on specific risk genes, the first attempts have been made to identify the pathways that they influence. Using data from four of the GWAS, Nurnberger et al. [21] reported six pathways that showed replicable association with $\mathrm{BD}$, involving glutamate and calcium signalling, second messengers, and hormones. Together, these findings support the possibility that $\mathrm{BD}$ is, at least in part, an ion channelopathy [22], in which aberrant calcium signalling is important [23].

\section{Glossary}

Affect(ive): essentially synonymous with mood. BD is sometimes known as 'bipolar affective disorder'.

Bipolar I disorder: the 'textbook' form of the disorder, with full manic episodes.

Bipolar II disorder: a form of the disorder in which manic episodes are milder or briefer, and depression predominates.

Bipolar spectrum disorder: includes bipolar I and II, and other cases not meeting criteria for those disorders.

Cyclothymia: a personality type characterised by ups and downs of mood, but lacking the severity or functional impact of BD.

DSM-5: the fifth edition of the Diagnostic and Statistical Manual of Mental Disorders. Includes formal diagnostic criteria for BP. Published by the American Psychiatric Association in 2013 and widely used in research.

Euthymia (or euthymic): normal mood, neither manic nor depressed. ICD-10: the tenth edition of the International Classification of Mental and Behavioural Disorders was published by the World Health Organisation in 1992. ICD-10 describes the clinical features of $\mathrm{BD}$ but is rarely used in research since DSM- 5 criteria are operationalised and more detailed. ICD-11 will be released in 2018.

Manic depression (or manic depressive psychosis): an older term for BD.

Mixed affective states: when depressive and manic features are present simultaneously.

Psychotic BD: a severe form of BD, in which psychotic symptoms (delusions and hallucinations) occur during manic and/or depressive episodes.

Rapid cycling: occurrence of four or more episodes (or mania or depression) within a year. 
Table 1. GWAS Hits for BD

\begin{tabular}{|l|l|}
\hline Locus & Implicated gene(s) \\
\hline $2 \mathrm{q} 11.2$ & LMAN2L \\
\hline $2 \mathrm{q} 32.1$ & ZNF804A \\
\hline $3 \mathrm{p} 22.2$ & TRANK1 (LBA1) \\
\hline $5 \mathrm{p} 15.31$ & ADCY2 \\
\hline $6 \mathrm{q} 16.1$ & MIR2113, POU3F2 (OTF7) \\
\hline $6 \mathrm{q} 25.2$ & SYNE1 \\
\hline $7 \mathrm{p} 22.3$ & MAD1L1 \\
\hline $9 \mathrm{p} 21.3$ & Intergenic \\
\hline $10 \mathrm{q} 21.2$ & ANK3 \\
\hline $11 \mathrm{q} 14.1$ & TENM4 (ODZ4) \\
\hline $12 \mathrm{p} 13.3$ & CACNA1C \\
\hline $12 \mathrm{q} 13.1$ & DDN \\
\hline $17 \mathrm{q} 12$ & ERBB2 \\
\hline
\end{tabular}

aData from $[98,117]$

\section{Calcium Signalling in Bipolar Disorder: Linking Genetics, Pathophysiology,} and Therapeutics

Calcium dysregulation has long been implicated in $\mathrm{BD}$, based primarily on ex vivo studies in cells taken from patients and controls. The findings are disparate, but on balance indicate that measures of intracellular calcium signalling are increased in BD, especially after stimulation (reviewed in [23,24]). The abnormalities appear largely independent of current mood state (i.e., they are trait rather than state related). Moreover, they are attenuated by lithium, which is used in the treatment of the disorder (Box 1). Despite the many uncertainties, these findings led to Ltype voltage-gated calcium channel (VGCC) antagonists, with existing indications in angina and hypertension, being evaluated for the treatment of BD [25]. Antiepileptic drugs, such as pregabalin, which act via VGCC $\alpha_{2} \delta$ subunits (Box 2) have also been tested [26], and lamotrigine, another antiepileptic drug that may block calcium channels, among its various actions [27], is an effective treatment for bipolar depression [28].

The results of the recent genomic studies strongly suggest that the involvement of calcium signalling in BD is at least partly causal [29], and have rekindled attempts to explain more precisely the nature of the alterations, not least because this may provide clues to moreeffective and tolerable drug strategies to normalise them [30]. However, the discovery of genetic variants is only the first step, and provides many more questions than answers. Calcium signalling offers an informative exemplar to highlight the opportunities and complexities associated with moving from psychiatric genomic discoveries to pathophysiological insights and therapeutic advances [31,32].

Genomic data provide a starting point to identify the molecules involved in the core 'calcium pathophysiology' of BD. They focus attention on the VGCCs, especially of the L-type, and their accessory subunits (encoded by the CACNx genes; Box 2). As indicated above, the best evidence is for CACNA1C (encoding the $\alpha 1$ subunit of $\mathrm{Ca}_{v}$ 1.2), but pathway analysis also suggests a role for CACNA1D and CACNB3 [29,33], and other VGCC genes are implicated in 


\section{Box 2. VGCC Genes, Their Isoforms, and Relevance in Bipolar Disorder}

Identifying the specific VGCCs most relevant for $\mathrm{BD}$ is a significant challenge, because their genes give rise to a vast diversity of functional channels (named $\mathrm{Ca}_{v}$ channels) $[96,97]$. VGCCs comprise multiple subunits, each encoded by one of a subfamily of separate genes. The properties of the ten distinct $\alpha 1$ subunits (encoded by the CACNA1- gene family) depend on the accessory subunits to which it is bound (Figure I). The main accessory subunits are the $\beta$ (encoded by CACNB1-4) and $\alpha_{2} \delta$ (encoded by CACNA2D1-4) subunits, which are obligate in most cases [98]. Current VGCC antagonists block the L-type channels; the anticonvulsant/analgesic drugs pregabalin and gabapentin are $\alpha_{2} \delta$ ligands [30].

Further channel diversity arises because each gene gives rise to multiple isoforms. The human CACNA1C mRNA has at least 50 exons and over 40 predicted isoforms (arising from transcriptional and splicing mechanisms). CACNA1C splicing gives rise to channel isoforms that are differentially expressed in brain compared with heart, and which differ in their biophysical properties, including voltage-gating characteristics [97]. Another feature affected by splicing is the isoform sensitivity to existing VGCC antagonists [98]. This suggests that it might be possible to selectively target splice variants that mediate disease risk and/or are preferentially expressed in the brain, compared with peripheral tissues (particularly the cardiovascular system, where VGCCs are also abundant), thereby maximising their therapeutic potential and tolerability in $\mathrm{BD}$ [25].

Given these considerations, defining the repertoire of VGCCs present in different human tissues is important, as is identifying which ones are impacted by the $\mathrm{BD}$-associated risk variants or by $\mathrm{BD}$ itself. However, information on the transcript diversity of human VGCC subunits is sparse, particularly in brain. Furthermore, because VGCC subunit genes are large (full-length CACNA1C mRNA, for instance, is over $10 \mathrm{~kb}$ long), the transcript structure of most isoforms remains unclear. Characterising the profile of full-length VGCCs isoforms in the human brain, compared with other tissues, and assessing which are altered in association with genetic risk for $\mathrm{BD}$, are critical first steps in translating the VGCC genomic findings into pathophysiological insights and novel treatment targets. The availability of large, highquality human postmortem brain series and technological advances in the field of RNA sequencing make this goal achievable.

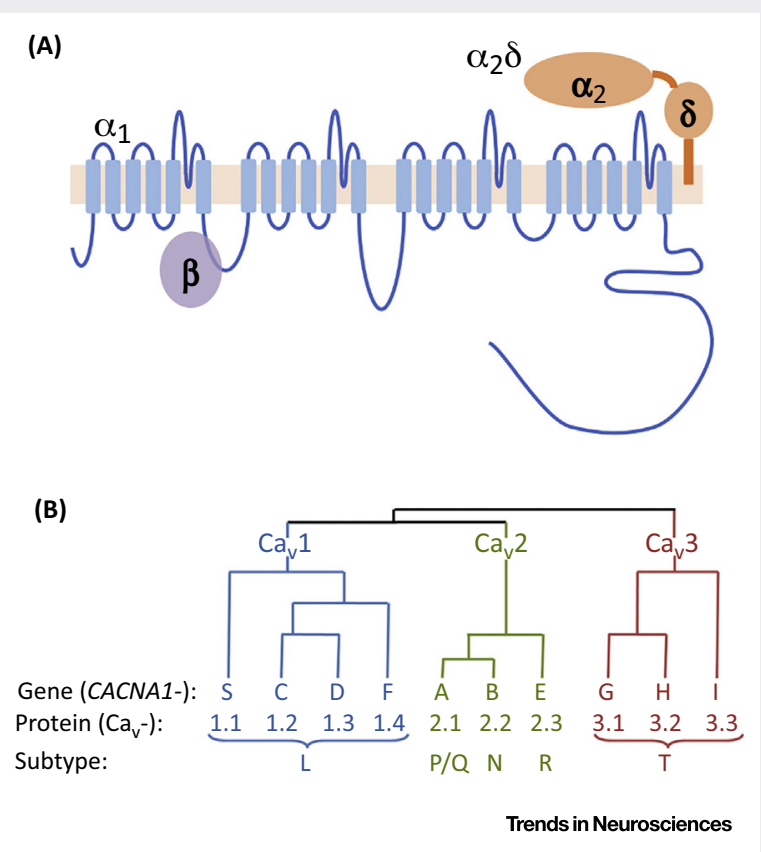

Figure I. The Voltage-Gated Calcium Channel (VGCC) Family of Proteins. (A) Structure of VGCCs, showing the transmembrane topology of the $\alpha 1$ subunit, its long intracellular $C$ terminus and interactions with accessory ( $\beta$ and $\alpha_{2} \delta$ ) subunits. (B) Dendrogram and nomenclature of the VGCC family. 
$\mathrm{BD}$ by rare variant studies [13]. Of note, apart from BD, CACNA1C is associated with schizophrenia [34] and major depression [35], and CACNB2 confers susceptibility to multiple psychiatric disorders [17]. Involvement of VGCC genes has also been reported in large-scale genomic studies of BD-relevant phenotypes, such as working memory performance and the associated patterns of brain activation [36], as well as in general cognitive functioning [37]. There are also smaller candidate gene studies that suggest effects of CACNA1C genetic variation on brain imaging phenotypes [38,39] and on cognitive domains, such as reward responsiveness [40].

Identification of the molecular basis for disease associations is a key step in understanding the mechanisms linking VGCC genes with BD. The VGCC loci revealed by GWAS are noncoding, and while large-scale exome studies may identify rare variants that disrupt the coding sequence of VGCCs, it is unlikely that the BD-associated GWAS loci tag as-yet-unidentified, diseasecausing mutations. Instead, they probably act by influencing aspects of gene expression, including methylation [41], alternative promoter usage, and RNA splicing [42]. In the case of CACNA1C, the index risk polymorphism for $\mathrm{BD}$ ( $\mathrm{rs} 1006737$ ) is located in the third intron. On balance, the available data indicate that the risk allele is associated with enhanced expression and activity of the gene product [43,44], but there are conflicting findings [45,46], precluding firm conclusions. Some of the variability in these results may be due to differences in the effect of rs1006737 on CACNA1C expression between brain regions. Inconsistencies may also result from the risk single nucleotide polymorphism (SNP) differentially altering the abundance of particular splice variants, as has been observed for other BD-relevant genes, including ANK3 [47] and ZNF804A [48]. In the case of $A N K 3$, there is evidence that the shift in isoform ratios has functional consequences for neuronal physiology [49]. Efforts to identify and understand whether altered splicing is also relevant for CACNA1C and other VGCCs are hampered by the limited information about their isoform profile in the human brain (Box 2). This information is critical since splicing patterns are poorly conserved and the brain shows one of the greatest diversities of alternative splicing [50], meaning that the current data, which pertain to other species and tissues, are insufficient.

While the identification of specific VGCC subunits and isoforms that mediate BD risk is important, better understanding of the underlying biology is also crucial. This requires the use of appropriate cellular and animal model systems, as well as in vivo approaches in humans. For cellular analyses, in addition to standard cell lines, which are useful for studying the function of individual genes, induced pluripotent stem cells (iPSCs) may prove valuable. Indeed, iPSCderived neurons have already provided intriguing data to support the presence of cellular BDrelated phenotypes, including alterations in calcium signalling (Box 3).

The molecular findings can also help guide the development of rodent models overexpressing or lacking specific VGCC genes [51-53] or splice variants thereof [54], in which their functional impact can be studied in the intact animal. For example, embryonic deletion of Cacna1c from forebrain glutamatergic neurons in mice produced BD-relevant behavioural and cognitive effects and an increased susceptibility to stress, whereas the same deletion during adulthood caused a lesser and, in some instances, opposite phenotype [51]. This effect of developmental stage on the phenotype is intriguing, given the characteristic early adulthood age of BD onset, and its childhood antecedents (Box 1). In a separate study, the phenotype of Cacna1c-deficient mice could be rescued using a small-molecule inhibitor of the translation initiation factor elF2 $\alpha$ [52], giving clues as to the possible intervening biochemical mechanisms.

In parallel with these various genetically driven approaches, pharmacological investigations of VGCCs in humans are possible because of the existing L-type VGCC antagonists, which can 


\section{Box 3. Stem Cells and the Calcium Pathophysiology of Bipolar Disorder}

The potential of iPSCs for studying cellular phenotypes of psychiatric disorders such as BD is considerable, and the approach has already been exploited in several studies. These have been of three designs: comparison of BD with healthy patients; contrasting lithium-responsive versus lithium-unresponsive BD cases; or evaluating the effect of BD risk genotypes (reviewed in [99]).

Mertens et al. [100] generated dentate gyrus-like neurons and showed that cells from patients with BD were hyperexcitable, with differences in several electrophysiological and transcriptional parameters; moreover, the excitability was normalised by lithium, but only in cells derived from patients who had responded clinically to the drug. Notably, these findings were largely replicated in a separate cohort and using a different methodology [101]. Taking a genetic strategy, Yoshimizu et al. [44] made induced neurons from 24 people, genotyped for the CACNA1C rs 1006737 polymorphism, and showed that $C A C N A 1 C$ gene expression and calcium current density were greater in risk allele homozygotes compared with nonrisk carriers.

3D differentiation approaches are now being used to produce 'brain spheroids'. Although no studies of this kind have yet been reported using iPSCs from patients with BD, Birey et al. [102] reported that, intriguingly, spheroids made with iPSCs from patients with Timothy syndrome (caused by a gain-of-function coding CACNA1C mutation) exhibited interneuron migratory abnormalities that could be normalised by a VGCC antagonist. The finding draws attention to a possible role for early developmental events and specific interneuron populations in mediating the role of calcium signalling in the pathogenesis of BD. Studies using spheroids in BD may be valuable in helping identify circuit-level phenotypes, while CRISPR techniques to selectively manipulate disease-relevant VGCC isoforms and variants could aid the identification of the key molecular mechanisms.

These findings, and others (e.g., [103]), illustrate how iPSCs are providing new clues regarding the neuronal phenotypes of $\mathrm{BD}$, and support an involvement of calcium signalling in these processes. The results encourage further efforts to extend and scale up the work [104].

be used as experimental tools and for proof-of-principle studies. Their availability is a distinct advantage compared with most other genetically supported therapeutic targets in $\mathrm{BD}$, for which no such drugs are available. To date, the clinical trial data linking L-type VGCC blockade with therapeutic outcome in BD (and, indeed, their psychiatric effects more generally) are wholly inconclusive [25]. Hence, a priority is to investigate in detail the impact of brain-penetrant VGCC blockers on BD-relevant phenotypes, including detailed measures of mood, cognition, sleep, and brain activity [55], and with the incorporation of genotype as a factor [56]. The potential psychiatric effects of VGCC antagonists can also be assessed using routinely collected clinical data; for example, a study of electronic medical records in Scotland reported higher hospital admission rates for depression in patients given VGCCs compared with those prescribed other antihypertensives [57].

In summary, a range of methods are needed to make the most of the genomic discoveries in $\mathrm{BD}$, to understand their molecular mechanisms and implications for cellular and systems functioning, and to evaluate their therapeutic potential. The conceptual approach is illustrated in Figure 1 (Key Figure), which uses VGCCs as the exemplar.

\section{New Approaches to the Bipolar Phenotype}

\section{Digital Psychiatry}

Complementing the developments from genomics-driven discovery science, novel methods are being applied to characterise the BD phenotype, and thereby provide new perspectives on its key elements.

Conventional psychiatric assessments rely on cross-sectional, retrospective analysis of the pattern of symptoms over weeks or months. Being based on a patient's or informant's recall, this approach is subject to inherent biases and unreliability, especially when, as in BD, the key 


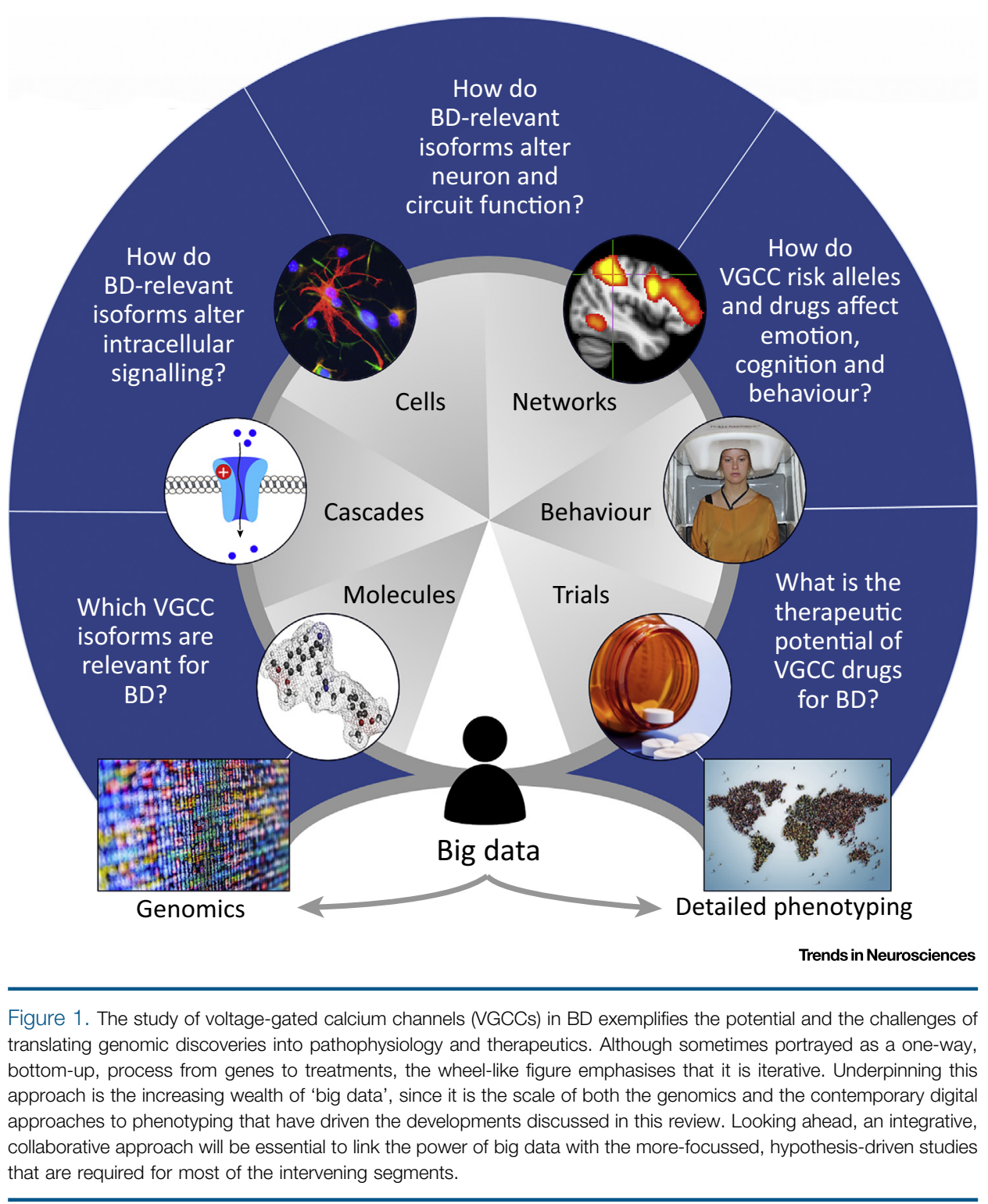

feature is not simply a symptom (mood), but its profile of variation over time. Several approaches have been used to try to improve the reliability and validity of BD assessments $[58,59]$. The main advances are emerging from developments in information technology, including the increasingly widespread use of connected and wearable devices: we are entering an era of 'digital phenotyping' in psychiatry [60], with BD, we would argue, at the forefront [61]. 
One early example is the True Colours platform, which allows patients to submit (by text, email, web, or app) their ratings of depression, mania, and other symptoms, in response to a weekly or daily prompt, resulting in a longitudinal and graphical representation of symptom course [62].

Importantly, smartphones and other devices allow the remote capture of not only contemporaneous self-reporting of symptoms, but also behavioural, cognitive and physiological measures, such as heart rate, activity, geolocation, speech, and environmental interactions [63-68] In turn, digital methods can facilitate a change from entirely symptom-based characterisation of psychiatric disorders, such as BD, towards a more multimodal, biologically informed one. Thus, they raise the prospect of more-objective, data-driven diagnoses, and ultimately personalised predictions of illness and treatment response. However, this promise has yet to be realised; the initial hype about digital phenotyping, including its application to BD, has been tempered by increasing awareness of the significant technical, analytical, and other issues involved (Box 4).

\section{Persistent Mood Instability}

Notwithstanding the many challenges, the longitudinal, long-term collection of data using digital approaches has already contributed to a renewed focus on the 'real-world' phenotype of BD. One example is the appreciation that many patients have chronic mood instability (also called affective lability), persisting during euthymia. This contrasts with the simplistic textbook description of BD as comprising periods of normal, stable mood in between the episodes of depression and mania (Box 1). Although this reality was already appreciated by experienced clinicians $[69,70]$, it is the use of digital methods that has led to a greater awareness of mood instability, which in turn has encouraged research to understand its origins and significance. Here, we briefly review some of these implications.

Mood (in)stability is a continuous variable, and is present to varying extents in healthy individuals $[71,72]$. Therefore, it is a useful phenotype for studies seeking to identify mechanisms underlying mood (dys)regulation per se, and a range of experimental approaches are being taken. For example, it can be viewed from a computational perspective, with models showing how mood

\section{Box 4. Digital and Mathematical Approaches to Bipolar Disorder}

Collection of multidimensional data, as in the remote monitoring of symptoms, behaviours, and physiology, has considerable potential in the study and treatment of $\mathrm{BD}$ and other disorders, but also throws up several significant challenges $[105,106]$.

First, there are many technical and practical issues to overcome, ranging from ensuring the compatibility of data collection between operating systems and software versions, to maintaining compliance over long time periods. There are also privacy, acceptability, and engagement issues raised by the recording and storage of personal data [107].

Second, the resulting data sets are large and complex, and the extraction, analysis, and interpretation of information are not straightforward [108]. One key question to consider is the mathematical approach chosen for analysing the data. Options include linear and nonlinear time series methods [109-111] as well as more-flexible and advanced techniques, such as relaxation oscillator frameworks [112] and machine-learning methods [90]. Another mathematical technique of interest is rough paths theory [113]. By taking account of lead-lag relationships, rough paths can reduce time-stamped data sets from complex interacting nonlinear systems to their critical information content or 'signature'. This transformation provides a structured sequence of low-dimensional summaries of the primary data that completely characterise their complexity but are amenable to linear analysis. This greatly facilitates efforts to use the data for classification and prediction. For example, a rough path signature, based on daily measures of mood instability, can differentiate BD from borderline personality disorder [114].

Progress in this developing field will need to be interdisciplinary, combining advanced mathematical analyses with digital phenotyping technologies that produce robust data and that are sufficiently acceptable, and rewarding, to patients to secure their long-term engagement $[115,116]$. 
instability interacts with reward sensitivity to alter performance [73]. One can also ask how mood instability relates to variation in neural activity across a range of temporal resolutions, using functional MRI [74] and magnetoencephalography [75]. In addition, mathematical analyses, such as those outlined in Box 4, can help explain the relationships between fluctuations in mood and other parameters, ranging from environmental exposures to behavioural variables $[66,67]$.

Mood instability is prominent not only in $\mathrm{BD}$, but also in several other psychiatric disorders, such as attention deficit disorder, borderline personality disorder, and schizophrenia [76,77]. It is therefore a trans-diagnostic construct, compatible with the NIMH Research Domain Criteria project [78]. Thus, understanding the origins and mechanisms of mood instability, and the biological and behavioural sequelae of interventions which modify it - is therefore likely to have value beyond $\mathrm{BD}$. Indeed, mood instability is a moderately heritable trait in the general population, and the first genome-wide significant loci have been reported [79]. Apart from studying the implicated molecules and pathways in their own right, it will be of interest to investigate the extent to which the risk loci for mood instability overlap with those for BD specifically, and other disorders involving mood instability. Moreover, the specific characteristics of mood instability (e.g., its frequency, amplitude, or impact on behaviour), while partially overlapping, may differ sufficiently between disorders [80] to help gain traction on the underlying neural mechanisms, providing further clues to the biological bases of these illnesses and leading to refinements in classification.

Finally, mood instability has potential immediate value as a therapeutic target. First, it may provide a way to screen novel treatments for BD more rapidly and cost-effectively than conventional clinical trials, in which treatment or prevention of depressive or manic episodes are the usual outcomes, requiring prolonged periods of observation. In this sense, early mood stabilisation could prove to predict therapeutic efficacy in the way that rapid effects on emotional processing predict subsequent therapeutic response in unipolar depression [81]. Moreover, the rapid effects of antidepressants on emotional processing are seen in euthymic subjects as well as in those with depression; similarly, new treatments for BD, such as novel VGCC-acting drugs, could be screened in patients who have unstable mood but who do not have a formal diagnosis. Second, stabilisation of mood may be beneficial in its own right, above and beyond simply preventing clinical episodes of depression and mania, because mood instability independently predicts poor functional recovery in BD, and worse outcomes of various kinds [76,82-84]. In addition, since mood instability often occurs before the onset of BD $[85,86]$, such treatments might have a preventative role.

In summary, the emergence of persistent mood instability as a construct illustrates how digital methods can highlight neglected or hard-to-characterise psychiatric phenomena, and stimulate a range of studies into their origins, mechanisms, and therapeutic implications. Comparable approaches are being taken to other components of $\mathrm{BD}$, such as circadian dysregulation and reward sensitivity. Although the work is at an early stage, it is likely ultimately to alter the view of the core phenotype of $\mathrm{BD}$, with a greater role for biological and quantitative data in $\mathrm{BD}$ diagnosis, prognosis, and management.

\section{Concluding Remarks}

Our understanding of BD remains frustratingly limited. It continues to be a descriptive syndrome, since we lack sufficient knowledge to allow its characterisation or conceptualisation based on aetiology or mechanism. Certainly, many questions remain (see Outstanding Questions). However, there are reasons for optimism. First, the discovery of some of the BD risk
Outstanding Questions

What is the overall genetic architecture of $\mathrm{BD}$ ? What are the main pathways impacted by the genes?

How will genetics alter the diagnosis of $\mathrm{BD}$ and its relationship to other disorders?

What are the causes and mechanisms of altered calcium signalling in $\mathrm{BD}$ ?

What is the core neurobiology of $\mathrm{BD}$, at systems and cellular levels?

Will genetic findings and novel phenotyping lead to improved therapies for $\mathrm{BD}$ ? 
genes has the potential to revolutionise our understanding of its pathogenesis and neurobiology. Second, the use of digital technologies and remote sensors, coupled with advanced analyses of the resulting data, is already allowing a more-quantitative, longitudinal approach to the BD phenotype. This raises the potential for better prediction of an individual's clinical course, and also provides a more-sophisticated phenotype for behavioural and biological studies. In both of these domains, a common feature is the increasing importance of 'big data', whether in terms of the genomic studies, or the multidimensional data streams captured by digital devices. Third, although not discussed here, structural and functional brain imaging is helping to identify the key neural circuits of BD and may also have diagnostic and prognostic value [2-4,87-90].

The ultimate goal of these contemporary approaches is to allow a more scientifically informed, evidence-based approach to how BD is classified, measured, and treated [91]. Possible changes include redrawing or removing the diagnostic boundaries between BD and other disorders involving lability of mood, emotion, and behaviour; the inclusion of behavioural and physiological correlates of mood and mood instability (or other symptoms) into clinical practice; a focus on identifying interventions that can stabilise mood independent of the underlying diagnosis; and new, genetically informed treatments, such as those targeting VGCCs.

It is not always appreciated that BD causes a global health burden comparable with that of schizophrenia [92], yet it has attracted considerably less research funding and interest from policy makers [93]. We would argue that, for various reasons, BD currently has a greater potential for transformative advances. More generally, BD is a useful case study for illustrating how psychiatric disorders are belatedly embarking on the journey from being descriptive syndromes towards more neurobiologically grounded, quantitative, and digital phenotypes [94]. Despite the many difficulties this process entails, it is not unreasonable to hope that it will be successful, and accompanied by the development of more rational, effective, and personalised treatments.

\section{Acknowledgments}

J.R.G. is a National Institute of Health Research (NIHR) Senior Investigator. E.M.T. is a Royal Society University Research Fellow. Research supported by Wellcome Trust strategic awards102616 and 09846, Medical Research Council grant P026028/1, and by the Oxford Health NIHR Biomedical Research Centre. The views expressed are those of the authors and not necessarily those of the National Health Service, NIHR or the Department of Health. We thank Kia Nobre for the idea for Figure 1 and Ruth Abrahams and Noel Buckley for supplying images. We apologise to the many authors whose studies could not be cited due to reference restrictions.

\section{References}

1. Craddock, N. and Sklar, P. (2013) Genetics of bipolar disorder. Lancet 381, 1654-1662

2. Phillips, M.L. and Swartz, H.A. (2014) A critical appraisal of neuroimaging studies of bipolar disorder: toward a new conceptualization of underlying neural circuitry and a road map for future research. Am. J. Psychiatry 171, 829-843

3. Wise, T. et al. (2016) Voxel-based meta-analytical evidence of structural disconnectivity in major depression and bipolar disorder. Biol. Psychiatry 79, 293-302

4. Hibar, D.P. et al. (2017) Cortical abnormalities in bipolar disorder: an MRI analysis of 6503 individuals from the ENIGMA Bipolar Disorder Working Group. Mol. Psychiatry Published online May 2, 2017. http://dx.doi.org/10.1038/mp.2017.73

5. Brown, N.C. et al. (2014) An updated meta-analysis of oxidative stress markers in bipolar disorder. Psychiatry Res. 218, $61-68$

6. Andreazza, A.C. et al. (2017) Bipolar disorder as a mitochondrial disease. Biol. Psychiatry Published online September 28, 2017. http://dx.doi.org/10.1016/j.biopsych.2017.09.018

7. Goldsmith, D.R. et al. (2016) A meta-analysis of blood cytokine network alterations in psychiatric patients: comparisons between schizophrenia, bipolar disorder and depression. Mol. Psychiatry 21, 1696-1709

8. Melo, M.C.A. et al. (2017) Chronotype and circadian rhythm in bipolar disorder: a systematic review. Sleep Med. Rev. 34, 46-58

9. Ashok, A.H. et al. (2017) The dopamine hypothesis of bipolar affective disorder: the state of the art and implications for treatment. Mol. Psychiatry 22, 666-679

10. Grande, I. et al. (2016) Bipolar disorder. Lancet 387, 1561-1572

11. Harrison, P.J. et al. (2016) Innovative approaches to bipolar disorder and its treatment. Ann. N.Y. Acad. Sci. 1366, 76-89 
12. Goes, F.S. et al. (2016) Exome sequencing of familial bipolar disorder. JAMA Psychiatry 73, 590-597

13. Ament, S.A. et al. (2015) Rare variants in neuronal excitability genes influence risk for bipolar disorder. Proc. Natl. Acad. Sci. U. S. A. 112, 3576-3581

14. Kataoka, M. et al. (2016) Exome sequencing for bipolar disorder points to roles of de novo loss-of-function and protein-altering mutations. Mol. Psychiatry 21, 885-893

15. Charney, A.W. et al. (2017) Evidence for genetic heterogeneity between clinical subtypes of bipolar disorder. Transl. Psychiatry 7, e993

16. O'Donovan, M.C. and Owen, M.J. (2016) The implications of the shared genetics of psychiatric disorders. Nat. Med. 22, 1214-1219

17. Cross-Disorder Group of the Psychiatric Genomics Consortium (2013) Genetic relationship between five psychiatric disorders estimated from genome-wide SNPs. Nat. Genet. 45, 984-994

18. Fears, S.C. et al. (2014) Multisystem component phenotypes of bipolar disorder for genetic investigations of extended pedigrees. JAMA Psychiatry 71, 375-387

19. Pagani, L. et al. (2016) Genetic contributions to circadian activity rhythm and sleep pattern phenotypes in pedigrees segregating for severe bipolar disorder. Proc. Natl. Acad. Sci. U. S. A. 113, E754-E761

20. Green, E.K. et al. (2016) Copy number variation in bipolar disorder. Mol. Psychiatry 21, 89-93

21. Nurnberger, J.I. et al. (2014) Identification of pathways for bipolar disorder: a meta-analysis. JAMA Psychiatry 71, 657-664

22. Gargus, J.J. (2006) Ion channel functional candidate genes in multigenic neuropsychiatric disease. Biol. Psychiatry 60, 177-185

23. Berridge, M.J. (2014) Calcium signalling and psychiatric disease: bipolar disorder and schizophrenia. Cell Tissue Res. 357, 477-492

24. Warsh, J.J. (2004) Role of intracellular calcium signalling in the pathophysiology and pharmacotherapy of bipolar disorder: current status. Clin. Neurosci. Res. 4, 201-213

25. Cipriani, A. et al. (2016) A systematic review of calcium channel antagonists in bipolar disorder and some considerations for their future development. Mol. Psychiatry 21, 1324-1332

26. Houghton, K.T. et al. (2017) Biological rationale and potential clinical use of gabapentin and pregabalin in bipolar disorder, insomnia and anxiety: protocol for a systematic review and meta-analysis. BMJ Open 7, e013433

27. Ketter, T.A. et al. (2003) Potential mechanisms of action of lamotrigine in the treatment of bipolar disorders. J. Clin. Psychopharmacol. 23, 484-495

28. Geddes, J.R. et al. (2016) Comparative evaluation of quetiapine plus lamotrigine combination versus quetiapine monotherapy (and folic acid versus placebo) in bipolar depression (CEQUEL): a $2 \times 2$ factorial randomised trial. Lancet Psychiatry 3, 31-39

29. Heyes, S. et al. (2015) Genetic disruption of voltage-gated calcium channels in psychiatric and neurological disorders. Prog. Neurobiol. 134, 36-54

30. Zamponi, G.W. et al. (2015) The physiology, pathology, and pharmacology of voltage-gated calcium channels and their future therapeutic potential. Pharmacol. Rev. 67, 821-870

31. Harrison, P.J. (2015) Recent genetic findings in schizophrenia and their therapeutic relevance. J. Psychopharmacol. 29, 85-96

32. Breen, G. et al. (2016) Translating genome-wide association findings into new therapies for psychiatry. Nat. Rev. Neurosci. 19, 1392-1396

33. Psychiatric GWAS Consortium Bipolar Disorder Working Group (2011) Large-scale genome-wide association analysis of bipolar disorder identifies a new susceptibility locus near ODZ4. Nat. Genet. 43, 977-983

34. Schizophrenia Working Group of the Psychiatric Genomics Consortium (2014) Biological insights from 108 schizophrenia-associated genetic loci. Nature 511, 421-427
35. Rao, S.Q. et al. (2016) Common variants in CACNA1C and MDD susceptibility: a comprehensive meta-analysis. Am. J. Med. Genet. Neuropsychiatr. Genet. 171, 896-903

36. Heck, A. et al. (2014) Converging genetic and functional brain imaging evidence links neuronal excitability to working memory, psychiatric disease, and brain activity. Neuron 81, 1203-1213

37. Smeland, O.B. et al. (2017) Identification of genetic loci jointly influencing schizophrenia risk and the cognitive traits of verbalnumerical reasoning, reaction time, and general cognitive function. JAMA Psychiatry 74, 1065-1075

38. Dixson, L. et al. (2014) Identification of gene ontologies linked to prefrontal-hippocampal functional coupling in the human brain. Proc. Natl. Acad. Sci. U. S. A. 111, 9657-9662

39. Ou, X. et al. (2015) CACNA1C rs1006737 genotype and bipolar disorder: focus on intermediate phenotypes and cardiovascula comorbidity. Neurosci. Biobehav. Rev. 55, 198-201

40. Lancaster, T.M. et al. (2014) CACNA1C risk variant affects reward responsiveness in healthy individuals. Transl. Psychiatry 4, e461

41. Starnawska, A. et al. (2016) CACNA1C hypermethylation is associated with bipolar disorder. Transl. Psychiatry 6, e831

42. Xiao, X. et al. (2017) Molecular mechanisms underlying noncoding risk variations in psychiatric genetic studies. Mol. Psychiatry 22, 497-511

43. Bigos, K.L. et al. (2010) Genetic variation in CACNA1C affects brain circuitries related to mental illness. Arch. Gen. Psychiatry 67, 939-945

44. Yoshimizu, T. et al. (2015) Functional implications of a psychiatric risk variant within $C A C N A 1 C$ in induced human neurons. $M O$ Psychiatry 20, 162-169

45. Gershon, E.S. et al. (2014) A rare mutation of CACNA1C in a patient with bipolar disorder, and decreased gene expression associated with a bipolar-associated common SNP of CACNA1C in brain. Mol. Psychiatry 19, 890-894

46. Eckart, N. et al. (2016) Functional characterization of schizophrenia-associated variation in CACNA1C. PLOS One 11, e0157086

47. Hughes, T. et al. (2016) A loss-of-function variant in a minor isoform of ANK3 protects against bipolar disorder and schizophrenia. Biol. Psychiatry 80, 323-330

48. Tao, R. et al. (2014) Expression of ZNF804A in human brain and alterations in schizophrenia, bipolar disorder, and major depres sive disorder: a novel transcript fetally regulated by the psychosis risk variant rs1344706. JAMA Psychiatry 71, 1112-1120

49. Lopez, A.Y. et al. (2017) Ankyrin-G isoform imbalance and interneuronopathy link epilepsy and bipolar disorder. Mol. Psychiatry 22, 1464-1472

50. Barbosa-Morais, N.J. et al. (2012) The evolutionary landscape of alternative splicing in vertebrate species. Science 338 1587-1593

51. Dedic, N. et al. (2017) Cross-disorder risk gene CACNA1C differentially modulates susceptibility to psychiatric disorders during development and adulthood. Mol. Psychiatry Published online July 11, 2017. http://dx.doi.org/10.1038/mp.2017.133

52. Kabir, Z.D. et al. (2017) Rescue of impaired sociability and anxiety-like behavior in adult cacna1c-deficient mice by pharmacologically targeting elF2 $\alpha$. Mol. Psychiatry 22, 1096-1109

53. Terrillion, C.E. et al. (2017) Decreased nucleus accumbens expression of psychiatric disorder risk gene Cacna1c promotes susceptibility to social stress. Int. J. Neuropsychopharmacol. 20, 428-433

54. Jiang, Y.-Q. et al. (2013) Spinal morphine but not ziconotide or gabapentin analgesia is affected by alternative splicing of voltage-gated calcium channel Ca(V)2.2 pre-mRNA. Mol. Pain 9, 67

55. Kabir, Z.D. et al. (2016) L-type $\mathrm{Ca}^{2+}$ channels in mood, cognition and addiction: integrating human and rodent studies with a focus on behavioural endophenotypes. J. Physiol. 594, $5823-5837$ 
56. Ostacher, M.J. et al. (2014) Pilot investigation of isradipine in the treatment of bipolar depression motivated by genome-wide association. Bipolar Disord. 16, 199-203

57. Boal, A.H. et al. (2016) Monotherapy with major antihypertensive drug classes and risk of hospital admissions for mood disorders. Hypertension 68, 1132-1138

58. Bauer, M. et al. (2004) Using technology to improve longitudinal studies: self-reporting with ChronoRecord in bipolar disorder. Bipolar Disord. 6, 67-74

59. Cochran, A.L. et al. (2016) Data-driven classification of bipolar I disorder from longitudinal course of mood. Transl. Psychiatry 6 , e912

60. Insel, T.R. (2017) Digital phenotyping. Technology for a new science of behavior. JAMA 318, 1215-1216

61. Nicholas, J. et al. (2015) Mobile apps for bipolar disorder: a systematic review of features and content quality. J. Med. Internet Res. 17, e198

62. McKnight, R.F. et al. (2017) Longitudinal mood monitoring in bipolar disorder: course of illness as revealed through a short messaging service. J. Affective Disord. 223, 139-145

63. Faurholt-Jepsen, M. et al. (2015) Smartphone data as an electronic biomarker of illness activity in bipolar disorder. Bipolar Disord. 17, 715-728

64. Faurholt-Jepsen, M. et al. (2016) Voice analysis as an objective marker in bipolar disorder. Transl. Psychiatry 6, e856

65. Mclntyre, R.S. et al. (2014) Advancing biomarker research: utilizing 'Big Data' approaches for the characterization and prevention of bipolar disorder. Bipolar Disord. 16, 531-547

66. Grunerbl, A. et al. (2015) Smartphone-based recognition of state and state changes in bipolar disorder patients. IEEE J. Biomed. Health Inform. 19, 140-147

67. Palmius, N. et al. (2017) Detecting bipolar depression from geographic location data. IEEE Trans. Biomed. Eng. 64, 1761-177

68. Shou, H. et al. (2017) Dysregulation of objectively assessed 24hour motor activity patterns as a potential marker for bipolar disorder: results of a community-based family study. Transl. Psychiatry 7, e1211

69. Mackinnon, D.F. and Pies, R. (2006) Affective instability as rapid cycling: theoretical and clinical implications for borderline personality and bipolar spectrum disorders. Bipolar Disord. 8, 1-14

70. Henry, C. et al. (2008) Affective lability and affect intensity as core dimensions of bipolar disorders during euthymic period. Psychiatr. Res. 159, 1-6

71. Broome, M. et al. (2015) Mood instability. Br. J. Psychiatry 207, 283-285

72. Marwaha, S. et al. (2012) The prevalence and clinical associations of mood instability in adults living in England: results from the Adult Psychiatric Morbidity Survey 2007. Psychiatr. Res. 205, 262-268

73. Mason, L. et al. (2017) Mood instability and reward dysregulation - a neurocomputational model of bipolar disorder. JAMA Psychiatry Published online October 11, 2017. http://dx.doi.org/ 10.1001/jamapsychiatry.2017.3163

74. Broome, M. et al. (2015) Neurobiological and behavioural studies of affective instability in clinical populations: a systematic review. Neurosci. Biobehav. Rev. 51, 243-254

75. Baker, A.P. et al. (2014) Fast transient networks in spontaneous human brain activity. eLife 3, e01867

76. Patel, R. et al. (2015) Mood instability is a common feature of mental health disorders and is associated with poor clinical outcomes. BMJ Open 5, e007504

77. Trull, Y.J. et al. (2015) Affective dynamics in psychopathology. Emotion Rev. 7, 355-361

78. Insel, T.R. (2014) The NIMH Research Domain Criteria (RDoC) project. Am. J. Psychiatry 177, 395-397

79. Ward, J. et al. Genome-wide analysis in UK Biobank identifies four loci associated with mood instability and genetic correlation with major depressive disorder, anxiety disorder and schizophrenia. Transl. Psychiatry (in press)

80. Tsanas, A. et al. (2016) Daily longitudinal self-monitoring of mood variability in bipolar disorder and borderline personality disorder. J. Affect. Dis. 205, 225-233

81. Harmer, C.J. et al. (2009) Why do antidepressants take so long to act? A cognitive neuropsychological model of antidepressan drug action. Br. J. Psychiatry 195, 102-108

82. Streijelowitz, S.A. et al. (2013) Mood instability and functiona recovery in bipolar disorders. Acta Psychiatr. Scand. 128, 194-202

83. Ducasse, D, et al, (2017) Affect lability predicts occurrence of suicidal ideation in bipolar patients: a two-year prospective study. Acta Psychiatr. Scand. 135, 460-469

84. Gershon, A. and Eidelman, P. (2015) Inter-episode affective intensity and instability: predictors of depression and functional impairment in bipolar disorder. J. Behav. Ther. Exp. Psychiatry $46,14-18$

85. Hafeman, D.M. et al. (2016) Toward the definition of a bipola prodrome: dimensional predictors of bipolar spectrum disorders in at-risk youths. Am. J. Psychiatry 173, 695-704

86. Van Meter, A.R. et al. (2016) The bipolar prodrome: metaanalysis of symptom prevalence prior to initial or recurrent mood episodes. J. Am. Acad. Child Adolesc. Psychiatry 55, 543-555

87. Meda, S.A. et al. (2014) Multivariate analysis reveals genetic associations of the resting default mode network in psychotic bipolar disorder and schizophrenia. Proc. Natl. Acad. Sci. U. S. A. 111, E2066-E2075

88. Savitz, J.B. et al. (2014) Neuropathological and neuromorphometric abnormalities in bipolar disorder: view from the media prefrontal cortical network. Neurosci. Biobehav. Rev. 42 , 132-147

89. Roberts, G. et al. (2017) Functional dysconnection of the inferio frontal gyrus in young people with bipolar disorder or at genetic high risk. Biol. Psychiatry 81, 718-727

90. Librenza-Garcia, D. et al. (2017) The impact of machine learning techniques in the study of bipolar disorder: a systematic review. Neurosci. Biobehav. Rev. 80, 538-554

91. Frank, E. et al. (2015) All the world's a (clinical) stage: rethinking bipolar disorder from a longitudinal perspective. Mol. Psychiatry 20, 23-31

92. Whiteford, H.A. et al. (2015) The global burden of mental, neurological and substance use disorders: an analysis from the Global Burden of Disease Study 2010. PLoS One 10 e0116820

93. Goodwin, G.M. and Geddes, J.R. (2007) What is the heartland of psychiatry? Br. J. Psychiatry 191, 189-191

94. Bhugra, D. et al. (2017) The WPA-Lancet Psychiatry Commission on the Future of Psychiatry. Lancet Psychiatry 4, 775-818

95. Harrison, P.J. et al. (2017) Bipolar disorder. In Shorter Oxford Textbook of Psychiatry (7th edn) (Harrison, P.J., ed.), pp. 233252, Oxford University Press

96. Dolphin, A.C. (2016) Voltage-gated calcium channels and their auxiliary subunits: physiology and pathophysiology and pharmacology. J. Physiol. 594, 5369-5390

97. Striessnig, J. et al. (2014) L-type $\mathrm{Ca}^{2+}$ channels in heart and brain. WIREs Membr. Transp. Signal. 3, 15-38

98. Hofmann, F. et al. (2014) L-type CaV1.2 channels: from in vitro findings to in vivo function. Physiol. Rev. 94, 303-326

99. Harrison, P.J. (2016) Molecular neurobiological clues to the pathogenesis of bipolar disorder. Curr. Opin. Neurobiol. 36, $1-6$

100. Mertens, J. et al. (2015) Differential responses to lithium in hyperexcitable neurons from patients with bipolar disorder. Nature 527, 95-99

101. Stern, S. et al. (2017) Neurons derived from patients with bipola disorder divide into intrinsically different sub-populations of neurons, predicting the patients' responsiveness to lithium. Mol. 
Psychiatry Published online February 28, 2017. http://dx.doi. org/10.1038/mp.2016.260

102. Birey, F. et al. (2017) Assembly of functionally integrated human forebrain spheroids. Nature 545, 54-59

103. Tobe, B.T.D. et al. (2017) Probing the lithium-response pathway in hiPSCs implicates the phosphoregulatory set-point for a cytoskeletal modulator in bipolar pathogenesis. Proc. Natl. Acad. Sci. U. S. A. 114, E4462-E4471

104. Harrison, P.J. et al. (2016) Reprogramming psychiatry: stem cells and bipolar disorder. Lancet 387, 823-825

105. Monteith, S. et al. (2015) Big data are coming to psychiatry - a general introduction. Int. J. Bipolar Disord. 3, 21

106. Monteith, S. et al. (2017) Big data for bipolar disorder. Int. J. Bipolar Disord. 4, 10

107. Bauer, M. et al. (2017) Ethical perspectives on recommending digital technology for patients with mental illness. Int. J. Bipolar Disord. 5, 6

108. Mclntosh, A.M. et al. (2016) Data science for mental health: a UK perspective on a global challenge. Lancet Psychiatry 3, 993-998

109. Moore, P.J. et al. (2014) Mood dynamics in bipolar disorder. Int. J. Bipolar Disord. 2, 11

110. Bonsall, M.B. et al. (2012) Nonlinear time-series approaches in characterizing mood stability and mood instability in bipolar disorder. Proc. R. Soc. B Biol. Sci. 279, 3632
111. Holmes, E.A. et al. (2016) Applications of time-series analysis to mood fluctuations in bipolar disorder to promote treatment innovation: a case series. Transl. Psychiatry 6, e720

112. Bonsall, M.B. et al. (2015) Bipolar disorder dynamics: affective instabilities, relaxation oscillations and noise. J. R. Soc. Interface 12, 0670

113. Hambly, B. and Lyons, T. (2010) Uniqueness for the signature of a path of bounded variation and the reduced path group. Ann Mathemat. 171, 109-167

114. Arribas, I.P. et al. (2017) A signature-based machine learning model for bipolar disorder and borderline personality disorder. arXiv 1707.07124

115. Bidargaddi, N. et al. (2017) Digital footprints. Mol. Psychiatry 22 164-169

116. Dubad, M. et al. (2017) A systematic review of the psychometric properties, usability and clinical impacts of mobile mood-monitoring applications in young people. Psychol. Med. Published online June 23, 2017. http://dx.doi.org/10.1017/ S0033291717001659

117. Hou, L.P. et al. (2016) Genome-wide association study of 40,000 individuals identifies two novel loci associated with bipoar disorder. Hum. Mol. Genet. 25, 3383-3394 